PROCEEDINGS OF THE

AMERICAN MATHEMATICAL SOCIETY

Volume 138, Number 11, November 2010, Pages 3997-4007

S 0002-9939(2010)10389-1

Article electronically published on May 19, 2010

\title{
TRANSLATION-INVARIANT MONOTONE SYSTEMS II: ALMOST PERIODIC/AUTOMORPHIC CASE
}

\author{
HONGXIAO HU AND JIFA JIANG
}

(Communicated by Yingfei Yi)

\begin{abstract}
This paper studies almost periodic/automorphic monotone systems with positive translation invariance via skew-product flows. It is proved that every bounded solution of such systems is asymptotically almost periodic/automorphic. Applications are made to a chemical reaction network, especially to enzymatic futile cycles with almost periodic/automorphic reaction coefficients.
\end{abstract}

\section{INTRODUCTION}

It is well-known that strongly monotone dynamical systems have the generic convergence property (see [13, 14, 15, 36, 37, 41]). A series of conditions have been found by different researchers to guarantee global convergence for monotone dynamical systems. For monotone semiflows or mappings, these conditions are orbital stability/fixed point stability [1, 2, 3, 42, 10, 20, 25, 26, 27, possessing a first integral or invariant function with positive gradient [32, 28, 22, 34, 7, 8, 18, 19, 45, 46], sublinearity [16, 17, 40, 44, 43, minimal equilibria [47, 12, respectively.

Recently Angeli and Sontag [4, 5] and Angeli, Leenheer and Sontag [6] have contributed a new type of global convergence condition, named positive translation invariance, which is motivated by a chemical reaction network. A standard form for representing (well-mixed and isothermal) chemical reactions by ordinary differential equations is

$$
\dot{S}=\Gamma R(S),
$$

evolving on the nonnegative orthant $\mathbb{R}_{+}^{m}$, where $S$ is an $m$-vector specifying the concentrations of $m$ chemical species, $\Gamma: \mathbb{R}^{n} \rightarrow \mathbb{R}^{m}$ is the stoichiometry matrix, and $R: \mathbb{R}_{+}^{m} \rightarrow \mathbb{R}^{n}$ is a function which provides the vector of reaction rates for any given vector of concentrations. Choosing $\sigma \in \mathbb{R}_{+}^{m}$ and using the reaction coordinates $x$,

$$
S=\sigma+\Gamma x,
$$

Received by the editors August 3, 2009 and, in revised form, January 17, 2010

2010 Mathematics Subject Classification. Primary 37B55, 37C65, 34C27, 92C45.

Key words and phrases. Positive translation invariance, monotonicity, almost periodicity/ automorphy, one-covering property.

The second author is supported partially by Chinese NNSF grant 10671143, Shanghai NSF grant 09ZR1423100, and Innovation Program of Shanghai Municipal Education Commission and RFDP, and is the author to whom correspondence should be addressed.

(C)2010 American Mathematical Society Reverts to public domain 28 years from publication 
instead of the traditional species coordinates $S$, Angeli and Sontag [4, 5] and Angeli, Leenheer and Sontag [6] have investigated the monotonicity and global behavior of systems in the reaction coordinates,

$$
\dot{x}=f_{\sigma}(x)=R(\sigma+\Gamma x)
$$

evolving on the state space

$$
X_{\sigma}=\left\{x \in \mathbb{R}^{n}: \sigma+\Gamma x \geq 0\right\} .
$$

Suppose that the matrix $\Gamma$ has rank exactly $n-1$ and its kernel is spanned by a strongly positive vector $v$. Then the state space is invariant with respect to translation by $v$, namely,

$$
x \in X_{\sigma} \Rightarrow x+\lambda v \in X_{\sigma}, \forall \lambda \in \mathbb{R},
$$

and the solution flow $\varphi_{t}(\xi)$ generated by (1.2) enjoys positive translation invariance:

$$
\varphi_{t}(\xi+\lambda v)=\varphi_{t}(\xi)+\lambda v, \forall \xi \in X_{\sigma}, \lambda \in \mathbb{R} .
$$

Angeli, Leenheer and Sontag [6] have shown that (1.2) is cooperative if and only if the corresponding $R$-graph is sign-consistent. Assuming that the positively translation-invariant system is strongly monotone and all solutions are bounded in a suitable sense, Angeli and Sontag [4] have verified that every solution is convergent to an equilibrium. We 31 have considered the autonomous/periodic monotone systems with positive translation invariance without the "strong monotonicity" assumption and have proved global convergence to equilibrium/periodic solutions.

All these conditions in strongly monotone dynamical systems have the following common characteristics: all forward orbits have some stability property in some sense (for example, as Lemma 2.3 given in 4]) and the equilibrium set is either unique, that is, globally attractive in this case, or a totally ordered curve, and every interior equilibrium in it attracts a codimension one manifold. But if one only assumes that the dynamical system is monotone without the stronger notion, the equilibrium set structure is more complicated. If one wishes to get a global convergence result under each of the above five conditions, he/she will meet difficulties during applying any known methods used in the strongly monotone case. As Sontag et al. pointed out in [11, p. 297], checking this condition in practice is often not so easy, or even worse: a system may be monotone, but fails to satisfy the stronger notion.

The second author has introduced the so-called Lyapunov integer-valued function to get rid of the irreducibility/strong monotonicity condition and has proved global convergence for various monotone systems. The idea is to compare each point in a given positive limit set to an equilibrium/fixed point which is the greatest among those equilibria/fixed points minus this positive limit set. Such an integer is defined to be the maximum number of different components between the greatest equilibrium/fixed point and points in the given positive limit set. To each positive limit set there corresponds such an integer, which is called a Lyapunov integer-valued function. Together with the stability property with induction, one can choose a sequence of positive limit sets in a given positive limit set such that the corresponding sequence of Lyapunov integer-valued functions is strictly decreasing if this given positive limit set is not a singleton. In this way, it was proved that an equilibrium is globally attractive if and only if every forward orbit has compact closure (see 21, 23, 30, 9]). This technique was further exploited in the multiple 
equilibria/fixed points case to prove global convergent results in monotone (without stronger notion) dynamical systems with every equilibrium/fixed point stable 25, 26, 27], sublinear nonlinearity [24] and possessing a positive gradient first integral [28].

Now this method has even been developed to study skew-product monotone semiflows without the stronger notion (see [29]). Using this technique on each fibre together with the theory on topological dynamical systems, it is shown that every omega limit set for uniformly stable monotone skew-product semiflows is topologically conjugate to the base space flow if the base space flow is minimal and distal and the state space has lattice structure with an additional precompact assumption. Applying this abstract result to various almost periodic differential equations, one gets that if each solution has the compact property and is uniformly stable, then every solution for systems considered is asymptotically almost periodic. Novo, Obaya and Sanz [35] have shown that the omega limit set is a uniformly stable minimal set which admits a fiber distal flow extension if its semiorbit is uniformly stable, which not only extends the classical extension result in Shen and Yi 39, but also drops the distal assumption in the abstract result in [29]. Later, together with their topological result with this Lyapunov integer-valued function method, they have investigated a series of functional differential equations with infinite delay and obtained one covering property of the base space (35, 33), some of which are even new in autonomous/periodic cases. The idea in [38 is also along these lines.

Motivated by the study of Angeli and Sontag 4, 5] and Angeli, Leenheer and Sontag [6] and our previous work [31, we shall investigate the nonautonomous chemical reaction network. Suppose that the reaction rates depend on time almost periodically/automorphically:

$$
\dot{S}=\Gamma R(t, S)
$$

where $R(t, S)$ is almost periodic/automorphic in $t$, all other variables and notation are exactly the same as in the autonomous case. Choosing $\sigma \in \mathbb{R}_{+}^{m}$ and using the reaction coordinates $x$ :

$$
S=\sigma+\Gamma x,
$$

we transform (1.5) into a system in the reaction coordinates:

$$
\dot{x}=f(t, x):=f_{\sigma}(t, x)=R(t, \sigma+\Gamma x)
$$

evolving on the state space

$$
X_{\sigma}=\left\{x \in \mathbb{R}^{n}: \sigma+\Gamma x \geq 0\right\} .
$$

Suppose that the matrix $\Gamma$ has rank exactly $n-1$ and its kernel is spanned by a strongly positive vector $v$. Then the state space is invariant with respect to translation by $v$, namely,

$$
x \in X_{\sigma} \Rightarrow x+\lambda v \in X_{\sigma}, \forall \lambda \in \mathbb{R} .
$$

Assume that the system (1.6) has the uniqueness property for the initial value problem. Let $\varphi(t, \xi, f)$ denote the solution of (1.6) passing through $\xi$ at $t=0$. Then it enjoys positive translation invariance:

$$
\varphi(t, \xi+\lambda v, f)=\varphi(t, \xi, f)+\lambda v, \forall \xi \in X_{\sigma}, \lambda \in \mathbb{R} .
$$

It is easy to see that the solution for every $g \in H(f)$ preserves the positive translation invariance property (1.8), where $H(f)$ is the hull generated by $f$. So the skew-product flow induced by the almost periodic/automorphic system (1.6) has 
positive translation invariance. The purpose of this paper is to prove that every omega limit set for a positively translation-invariant monotone skew-product is conjugate to its minimal base flow. The main tool used here is included in [29, 35, 39]. As an application, we apply our result to the enzymatic futile cycles:

$$
\begin{aligned}
& E+P \leftrightarrow C \rightarrow E+Q, \\
& F+Q \leftrightarrow D \rightarrow F+P,
\end{aligned}
$$

which is a model of the activation of a protein substrate $P$ by an enzyme $E$; $C$ is an intermediate complex, which dissociates either back into the original components or into a product (activated protein) $Q$ and the enzyme. The second reaction transforms $Q$ back into $P$ and is catalyzed by another enzyme $F$. The mass-action kinetics model takes the form of (1.5) in which $S=\left(S_{1}, S_{2}, S_{3}, S_{4}, S_{5}, S_{6}\right)^{\prime}$ denotes the concentration species of $(P, Q, E, F, C, D)^{\prime}$, the stoichiometry matrix

$$
\Gamma=\left(\begin{array}{cccc}
-1 & 0 & 0 & 1 \\
0 & 1 & -1 & 0 \\
-1 & 1 & 0 & 0 \\
0 & 0 & -1 & 1 \\
1 & -1 & 0 & 0 \\
0 & 0 & 1 & -1
\end{array}\right)
$$

and the reaction rates

$$
R(S)=\left(\begin{array}{c}
k_{1} S_{1} S_{3}-k_{2} S_{5} \\
k_{3} S_{5} \\
k_{4} S_{2} S_{4}-k_{5} S_{6} \\
k_{6} S_{6}
\end{array}\right)
$$

where $k_{i}$ is a nonnegative almost periodic/automorphic function for $i=1,2, \ldots, 6$. It is concluded that every solution of (1.5) in this case is asymptotically almost periodic/automorphic.

\section{DeFinitions AND PRELIMINARY RESUlts}

Denote by $\mathbb{R}, \mathbb{R}^{+}$all real numbers and nonnegative real numbers, respectively, and let $Y$ be a compact metric space. Recall that a continuous flow $\sigma: \mathbb{R} \times Y \rightarrow Y$ is said to be minimal if $Y$ contains no nonempty, proper, closed totally invariant subset. Throughout this paper, we always assume that the flow $(Y, \sigma, \mathbb{R})$ is minimal.

Let $\left(V_{i}, V_{i}^{+}\right), 1 \leq i \leq n$, be ordered Banach spaces with $\operatorname{Int}\left(V_{i}^{+}\right) \neq \emptyset$. Define

$$
V:=\prod_{k=1}^{n} V_{k}, \quad V^{+}:=\prod_{k=1}^{n} V_{i}^{+} .
$$

Then $\left(V, V^{+}\right)$is an ordered Banach space with $\operatorname{Int}\left(V^{+}\right)=\prod_{k=1}^{n} \operatorname{Int}\left(V_{i}^{+}\right) \neq \emptyset$. For each $1 \leq i \leq n$, let $Q_{i}: V \times Y \rightarrow V_{i}$ be the projection defined by $Q_{i}(x, y)=x_{i}$, and $Q: V \times Y \rightarrow Y$ be the projection defined by $Q(x, y)=y$, respectively.

For $x_{1}, x_{2} \in V$, we write $x_{1} \leq x_{2}$ if $x_{2}-x_{1} \in V^{+} ; x_{1}<x_{2}$ if $x_{2}-x_{1} \in V^{+} \backslash\{0\}$; $x_{1} \ll x_{2}$ if $x_{2}-x_{1} \in \operatorname{Int}\left(V^{+}\right)$. A vector $v$ is strongly positive if $v \gg 0$.

The state space $X \subset V$ is assumed to be invariant with respect to translation by a strongly positive vector $v$ :

$$
x \in X \Rightarrow x+\lambda v \in X, \forall \lambda \in \mathbb{R} .
$$


This implies that $X$ is order convex; that is, if $a, b \in X$ with $a<b$, then the segment $\{a+s(b-a): s \in[0,1]\}$ is contained in $X$. Our first assumption is

(A1) every compact subset in $X$ has both the greatest lower bound and the least upper bound.

Consider a continuous skew-product semiflow $\Pi: \mathbb{R}^{+} \times X \times Y \rightarrow X \times Y$ defined by

$$
\Pi(t, x, y)=(u(t, x, y), \sigma(t, y)), \forall(t, x, y) \in \mathbb{R}^{+} \times X \times Y .
$$

Our next assumption is

(A2) For every $(x, y) \in X \times Y$, there is a $t_{0}=t_{0}(x, y)$ such that $\left\{\Pi_{t}(x, y): t \geq t_{0}\right\}$ is precompact.

Definition 2.1. Let $\Pi_{t}$ be a skew-product semiflow on $X \times Y$. The forward orbit $\Pi_{t}\left(x_{0}, y_{0}\right), t \geq 0$, is said to be uniformly stable if for every $\epsilon>0$, there is a $\delta=\delta(\epsilon)>0$, called the modulus of uniform stability, such that

$$
d\left(\Pi\left(\tau+t, x, y_{0}\right), \Pi\left(\tau+t, x_{0}, y_{0}\right)\right)<\epsilon, \quad \forall t \geq 0
$$

whenever $\tau \geq 0$ and $d\left(u\left(\tau, x, y_{0}\right), u\left(\tau, x_{0}, y_{0}\right)\right)<\delta$.

The following result comes from [35, improving the result in [39, page 29].

Theorem 2.2. Let $\Pi_{t}$ be a skew-product semiflow on $X \times Y$. If a forward orbit $\Pi_{t}\left(x_{0}, y_{0}\right)$ is precompact and uniformly stable, then $\omega\left(x_{0}, y_{0}\right)$ admits a fiber distal flow extension which is minimal.

The third assumption is

(A3) Every forward orbit of the skew-product semiflow $\Pi_{t}$ is uniformly stable.

For skew-product semiflows, we always use the order relation on each fiber $Q^{-1}(y)$. We write $\left(x_{1}, y\right) \geq_{y}\left(>_{y}, \gg_{y}\right)\left(x_{2}, y\right)$ if $x_{1} \geq x_{2}\left(x_{1}>x_{2}, x_{1} \gg x_{2}\right)$. Without any confusion, we will drop the subscript " $y$ ".

Definition 2.3. A skew-product semiflow $\Pi_{t}$ on $X \times Y$ is said to be monotone (strictly monotone, strongly monotone) if

$$
\Pi_{t}\left(x_{1}, y\right) \leq(<, \ll) \Pi_{t}\left(x_{2}, y\right)
$$

whenever $t>0$ and $\left(x_{1}, y\right)<\left(x_{2}, y\right)$.

The final assumption is

(A4) The skew-product semiflow $\Pi_{t}$ is monotone, and for any two bounded full orbits $\Pi_{t}\left(x_{1}, y\right) \leq \Pi_{t}\left(x_{2}, y\right), \forall t \in \mathbb{R}$, there exists $t_{0}>0$ such that whenever $Q_{i} \circ \Pi_{s}\left(x_{1}, y\right)<Q_{i} \circ \Pi_{s}\left(x_{2}, y\right)$ holds for some $i$ and $s \in \mathbb{R}$, then $Q_{i} \circ$ $\Pi_{t}\left(x_{1}, y\right) \ll Q_{i} \circ \Pi_{t}\left(x_{2}, y\right)$ for all $t \geq s+t_{0}$.

With the help of Theorem 2.2, the main results in 29] are stated as follows.

Theorem 2.4. Assume that (A1)-(A4) hold. Then for any $\left(x_{0}, y_{0}\right) \in X \times Y$, $\left(\omega\left(x_{0}, y_{0}\right), \Pi_{t}\right)$ is conjugate to $\left(Y, \sigma_{t}\right)$, and $\lim _{t \rightarrow \infty}\left\|u\left(x_{0}, y_{0}, t\right)-u\left(x_{0}^{*}, y_{0}, t\right)\right\|=0$, where $\left(x_{0}^{*}, y_{0}\right)=\omega\left(x_{0}, y_{0}\right) \cap Q^{-1}\left(y_{0}\right)$.

We note that every $\omega\left(x_{0}, y_{0}\right)$ is a continuous equilibrium according to 35 . 


\section{The MAIN RESUlt AND ITS PROOF}

Recall that our state space $X$ is assumed to be invariant with respect to translation by a strongly positive vector $v$. In this section we fix such a strongly positive vector $v$ as a unit vector. Further we assume that the cone $V^{+}$is normal. This means that there exists a constant $k>0$ such that whenever $0 \leq x \leq y$, then $|x| \leq k|y|$. Define the $v$-norm by

$$
\|x\|_{v}=\inf \{t>0:-t v \leq x \leq t v\} .
$$

The norms $|$.$| and \|.\|_{v}$ are equivalent if the cone $V^{+}$is normal.

Definition 3.1. A skew product semiflow $\Pi_{t}$ is said to be positively translationinvariant with respect to $v$ if

$$
u(t, x+\lambda v, y)=u(t, x, y)+\lambda v, \forall(t, x, y, \lambda) \in \mathbb{R}^{+} \times X \times Y \times \mathbb{R} .
$$

Theorem 3.2. Let $\Pi_{t}$ be a skew-product semiflow on $X \times Y$. Assume that (A1), (A2) and (A4) hold. If $\Pi_{t}$ is positively translation-invariant with respect to $v$, then for any $\left(x_{0}, y_{0}\right) \in X \times Y,\left(\omega\left(x_{0}, y_{0}\right), \Pi_{t}\right)$ is conjugate to $\left(Y, \sigma_{t}\right)$, and $\lim _{t \rightarrow \infty} \| u\left(x_{0}, y_{0}, t\right)$ $-u\left(x_{0}^{*}, y_{0}, t\right) \|=0$, where $\left(x_{0}^{*}, y_{0}\right)=\omega\left(x_{0}, y_{0}\right) \cap Q^{-1}\left(y_{0}\right)$, and $\omega\left(x_{0}, y_{0}\right)$ is a continuous equilibrium for $\Pi_{t}$.

From Theorem 2.4, we know that in order to finish the proof of Theorem 3.2. we only have to check uniform stability for every forward orbit, which is implied by the following proposition.

Proposition 3.3. Suppose that $X$ is invariant with respect to translation by a strongly positive vector $v$ and $\Pi_{t}$ is positively translation-invariant with respect to $v$. Then every forward orbit of the skew-product semiflow $\Pi_{t}$ is uniformly stable.

Proof. Fix $\left(x_{0}, y_{0}\right) \in(X, Y)$. Then we shall prove that

$$
\left\|u\left(t, x, y_{0}\right)-u\left(t, x_{0}, y_{0}\right)\right\|_{v} \leq\left\|x-x_{0}\right\|_{v} \text {, for all } t>0 .
$$

By the definition of $v$-norm,

$$
-\left\|x-x_{0}\right\|_{v} v \leq x-x_{0} \leq\left\|x-x_{0}\right\|_{v} v, \text { for all } x, x_{0} \in X
$$

that is,

$$
x_{0}-\left\|x-x_{0}\right\|_{v} v \leq x \leq x_{0}+\left\|x-x_{0}\right\|_{v} v \text {, for all } x, x_{0} \in X .
$$

This inequality implies together with monotonicity and positive translation invariance that for all $x, x_{0} \in X, t>0$,

$$
u\left(t, x_{0}, y_{0}\right)-\left\|x-x_{0}\right\|_{v} v \leq u\left(t, x, y_{0}\right) \leq u\left(t, x_{0}, y_{0}\right)+\left\|x-x_{0}\right\|_{v} v .
$$

Equivalently, for all $x, x_{0} \in X, t>0$,

$$
-\left\|x-x_{0}\right\|_{v} v \leq u\left(t, x, y_{0}\right)-u\left(t, x_{0}, y_{0}\right) \leq\left\|x-x_{0}\right\|_{v} v .
$$

(3.2) immediately follows from (3.3) and the definition of $v$-norm.

By the cocyle property, we have that

$$
u(t+\tau, x, y)=u(t, u(\tau, x, y), y \cdot \tau) \text { for all } x, y \in X, t, \tau>0 .
$$

From this cocyle property together with (3.2), we conclude that

$$
\left\|u\left(t+\tau, x, y_{0}\right)-u\left(t+\tau, x_{0}, y_{0}\right)\right\|_{v} \leq\left\|u\left(\tau, x, y_{0}\right)-u\left(\tau, x_{0}, y_{0}\right)\right\|_{v} \text {, for all } t, \tau>0 \text {. }
$$


This proves that every forward orbit of $\Pi_{t}$ is uniformly stable in the order-norm. The normality of the cone $V^{+}$implies that every forward orbit of $\Pi_{t}$ is uniformly stable.

Remark 3.4. Angeli and Sontag [4, page 132, Lemma 2.3] have given a result on the stability of autonomous positively translation-invariant monotone systems. From what we have described the stability property in (3.2) is more concise and forthright than theirs. Actually, if we take $V: X \rightarrow \mathbb{R}^{+}$by $V(\xi)=|\xi|{ }_{v}$, then $V$ is a Lyapunov function, and (3.2) reads

$$
V\left(u\left(t, x, y_{0}\right)-u\left(t, x_{0}, y_{0}\right)\right) \leq V\left(x-x_{0}\right), \forall x, x_{0} \in X ; y_{0} \in Y ; t>0,
$$

which is more precise than [4, page 132, (6)].

\section{Application}

In this section, we apply our Theorem 3.2 to a nonautonomous chemical reaction network (1.5).

Firstly we assume that the reaction rate $R: \mathbb{R} \times \mathbb{R}_{+}^{m} \rightarrow \mathbb{R}^{n}$ is continuous, and for any compact subset $K \subset \mathbb{R}_{+}^{m}$, there exists a Lipschitz constant $L=L(K)$ such that $R$ satisfies the Lipschitz condition with respect to $S$ with Lipschitz constant $L$ on $\mathbb{R} \times K$. Secondly, we assume that $R$ is admissible. This means that for any compact subset $K \subset \mathbb{R}_{+}^{m}, R$ is bounded and uniformly continuous on $\mathbb{R} \times K$. Finally, we assume that $R$ is almost periodic/almost automorphic with respect to $t \in \mathbb{R}$ (we refer to [39] for the precise definition).

Let $S=\sigma+\Gamma x$ and $f(t, x):=R(t, \sigma+\Gamma x)$. The domain for $x$ is

$$
X=\left\{x \in \mathbb{R}^{n}: \sigma+\Gamma x \geq 0\right\} .
$$

Lemma 4.1. Suppose that $R$ satisfies all the conditions stated above. If every solution of (1.5) is defined for all $t \geq 0$ and the positive orthant $\mathbb{R}_{+}^{m}$ is positively invariant for (1.5), then $X_{\sigma}$ is invariant under (1.6); i.e., for any $x_{0} \in X_{\sigma}$, the solution $x\left(t ; x_{0}\right)$ of (1.6) with $x\left(0 ; x_{0}\right)=x_{0}$ belongs to $X_{\sigma}$ for $t \geq 0$.

The proof is the same as the one of Lemma 3.1 in Angeli and Sontag [4], so we omit it.

Suppose that the matrix $\Gamma$ has rank exactly $n-1$ and its kernel is spanned by a strongly positive unit vector $v$. Then the state space $X$ is invariant with respect to translation by $v$. The assumptions for $R$ above can be inherited by $f$. Thus the vector function generates a family $\left\{f_{\tau} \mid \tau \in \mathbb{R}\right\}$ in $C\left(\mathbb{R} \times X, \mathbb{R}^{n}\right)$, where $f_{\tau}(t, x):=f(t+\tau, x)(\tau \in \mathbb{R})$ denotes the time translation. Let $H(f)$, the hull of $f$, be the closure of $\left\{f_{\tau} \mid \tau \in \mathbb{R}\right\}$ in the compact open topology. By Ascoli's theorem, $H(f)$ is compact and in fact is metrizable. Moreover, the time translation $g . t \equiv g_{t}(g \in H(f))$ induces a nature flow $(H(f), \mathbb{R})$, which is almost periodic/almost automorphic minimal (see 39 for details).

Let $g \in H(f)$. Then there exists a sequence $\left\{\tau_{n}\right\}$ such that

$$
f\left(t+\tau_{n}, x\right) \rightarrow g(t, x) \text { as } n \rightarrow \infty
$$

uniformly on every compact subset $[a, b] \times K$. Thus there exists a vector function $Q: \mathbb{R} \times \mathbb{R}_{+}^{m} \rightarrow \mathbb{R}^{n}$ such that $g(t, x)=Q(t, \sigma+\Gamma x)$ and $Q$ inherits the properties in three assumptions on $R$.

For any $g \in H(f)$, let $u(t, x, g)$ be the solution of the system

$$
\dot{x}=g(t, x)=Q(t, \sigma+\Gamma x)
$$


passing through $x$ at $t=0$. Then $u(t, x, g)$ enjoys the positive translation invariance with $v \gg 0$. Define the skew-product flow $\Pi$ on $X \times H(f)$ by

$$
\Pi_{t}(x, g)=\left(u(t, x, g), g_{t}\right) \text { for }(t, x, g) \in \mathbb{R} \times X \times H(f) .
$$

Then the skew-product flow $\Pi$ is positively translation-invariant with respect to $v$.

Theorem 4.2. Suppose that the following conditions hold:

(i) the matrix $\Gamma$ has rank exactly $n-1$ whose kernel is spanned by a strongly positive vector $v$;

(ii) $\sigma \in \mathbb{R}_{+}^{m}$ is such that the system (1.6) is monotone;

(iii) all forward solutions of (1.6) are bounded.

Then every solution of (1.6) is asymptotically almost periodic/automorphic. The same result is true for (1.5).

This theorem can be applied to enzymatic futile cycles where all $k_{i}$ are nonnegative almost periodic/automorphic functions. For this system, the stoichiometry matrix and reaction rates are given in the introduction. Explicitly the system in the reaction coordinates is

$$
\dot{x}=f(t, x)=\left(\begin{array}{c}
k_{1}(t)\left(\sigma_{3}+x_{2}-x_{1}\right)\left(\sigma_{1}+x_{4}-x_{1}\right)-k_{2}(t)\left(\sigma_{5}+x_{1}-x_{2}\right) \\
k_{3}(t)\left(\sigma_{5}+x_{1}-x_{2}\right) \\
k_{4}(t)\left(\sigma_{4}+x_{4}-x_{3}\right)\left(\sigma_{2}+x_{2}-x_{3}\right)-k_{5}(t)\left(\sigma_{6}+x_{3}-x_{4}\right) \\
k_{6}(t)\left(\sigma_{6}+x_{3}-x_{4}\right)
\end{array}\right),
$$

where the state space

$$
\begin{gathered}
X=\left\{\left(x_{1}, x_{2}, x_{3}, x_{4}\right): \sigma_{1}+x_{4}-x_{1} \geq 0, \sigma_{2}+x_{2}-x_{3} \geq 0, \sigma_{3}+x_{2}-x_{1} \geq 0,\right. \\
\left.\sigma_{4}+x_{4}-x_{3} \geq 0, \sigma_{5}+x_{1}-x_{2} \geq 0, \sigma_{6}+x_{3}-x_{4}\right\},
\end{gathered}
$$

and $v=\frac{1}{4}(1,1,1,1)$, and $k_{i}(t)$ is either nonnegative almost periodic or nonnegative almost automorphic for $i=1,2, \ldots, 6$. Then every solution is either asymptotically almost periodic or asymptotically almost automorphic. For example, when $k_{i}(t)>0$ for each $i, \sigma_{3}>0, \sigma_{4}>0, \sigma_{j}=0, j \neq 3,4$, every solution is asymptotic to a constant in the line $L=\{(\lambda, \lambda, \lambda, \lambda): \lambda \in \mathbb{R}\}$. In this case, the skew product flow is strongly monotone.

Before finishing this paper, we artificially construct two examples to show the potential use of our abstract result.

Example 4.3. Consider the delay differential equations

$$
\begin{aligned}
& \dot{x}(t)=f\left(t, x_{t}\right) \\
& \quad=\left(\begin{array}{c}
k_{1}(t)\left(\sigma_{3}+x_{2}(t-\tau)-x_{1}(t)\right)\left(x_{4}(t-\tau)-x_{1}(t)\right)-k_{2}(t)\left(x_{1}(t)-x_{2}(t-\tau)\right) \\
k_{3}(t)\left(x_{1}(t-\tau)-x_{2}(t)\right) \\
k_{4}(t)\left(\sigma_{4}+x_{4}(t-\tau)-x_{3}(t)\right)\left(x_{2}(t-\tau)-x_{3}(t)\right)-k_{5}(t)\left(x_{3}(t)-x_{4}(t-\tau)\right) \\
k_{6}(t)\left(x_{3}(t-\tau)-x_{4}(t)\right)
\end{array}\right),
\end{aligned}
$$

where $k_{i}(t)>0$ is almost periodic/automorphic for each $i, \sigma_{3}>0, \sigma_{4}>0$. The system generates a skew-product semiflow $\Pi_{t}$ defined on $C([-\tau, 0], X) \times H(f)$, which is positively translation-invariant with respect to $v=\frac{1}{4}(1,1,1,1)$. Thus every solution in this example is asymptotic to a constant in $L$. 
Example 4.4. Consider the reaction-diffusion system

$$
\frac{\partial u}{\partial t}=\Delta u+f(t, u)=\Delta u+\left(\begin{array}{c}
k_{1}(t)\left(\sigma_{3}+u_{2}-u_{1}\right)\left(u_{4}-u_{1}\right)-k_{2}(t)\left(u_{1}-u_{2}\right) \\
k_{3}(t)\left(u_{1}-u_{2}\right) \\
k_{4}(t)\left(\sigma_{4}+u_{4}-u_{3}\right)\left(u_{2}-u_{3}\right)-k_{5}(t)\left(u_{3}-u_{4}\right) \\
k_{6}(t)\left(\left(u_{3}-u_{4}\right)\right)
\end{array}\right)
$$

with Neumann boundary condition on the smooth boundary of manifold $\Omega$, where $k_{i}(t)>0$ is almost periodic/automorphic for each $i, \sigma_{3}>0, \sigma_{4}>0$. The system generates a skew-product semiflow $\Pi_{t}$ defined on $C(\Omega, X) \times H(f)$, which is positively translation-invariant with respect to $v=\frac{1}{4}(1,1,1,1)$. Thus every solution in this example is asymptotic to a constant in $L$.

\section{REFERENCES}

1. N. D. Alikakos and P. Hess, On stabilization of discrete monotone dynamical systems, Israel J. Math., 59 (1987), 185-194. MR920081 (89h:47083)

2. N. D. Alikakos and P. Bates, Stabilization of solutions for a class of degenerate equations in divergence form in one space dimension, J. Differential Equations, 73 (1988), 363-393. MR.943947 (90g:35018)

3. N. D. Alikakos, P. Hess and H. Matano, Discrete order preserving semigroups and stability for periodic parabolic differential equations, J. Differential Equations, 82 (1989), 322-341. MR.1027972 (91i:35016)

4. D. Angeli and E. D. Sontag, Translation-invariant monotone systems, and a global convergence result for enzymatic futile cycles, Nonlinear Anal. Real World Appl., 9 (2008), 128-140. MR 2370168 (2008k:92019)

5. D. Angeli and E. D. Sontag, A note on monotone systems with positive translation invariance, In Control and Automation, 2006. MED '06. 14th Mediterranean Conference on, 28-30 June 2006, pages 1-6.

6. D. Angeli, P. De Leenheer and E. D. Sontag, On the structural monotonicity of chemical networks, in Proc. IEEE Conf. Decision and Control, San Diego, Dec. 2006, pages 7-12.

7. O. Arino, Monotone semi-flows which have a monotone first integral, in "Delay Differential Equations and Dynamical Systems", Lecture Notes in Math., Vol. 1475, Springer-Verlag, Berlin/Heidelberg, 1991, pp. 64-75. MR.1132019 (93a:34081)

8. O. Arino and E. Haourigui, On the asymptotic behavior of solutions of some delay differential systems which have a first integral, J. Math. Anal. Appl., 122 (1987), 36-46. MR874957 (88b:34115)

9. F. Cao and J. Jiang, On the global attractivity of monotone random dynamical systems, Proc. Amer. Math. Soc., 138 (2010), 891-898.

10. E. N. Dancer and P. Hess, Stability of fixed points for order-preserving discrete-time dynamical systems, J. Reine Angew. Math., 419 (1991), 125-139. MR1116922 (92i:47088)

11. P. De Leenheer, D. Angeli and E. D. Sontag, Monotone chemical reaction networks, J. Math. Chemistry, 41 (2007), 295-314. MR2343862 (2009c:92041)

12. J. R. Haddock, M. N. Nkashama and J. Wu, Asymptotic constancy for pseudomonotone dynamical systems on function spaces, J. Differential Equations, 100(1992), 292-311. MR:1194812 (94b:34096)

13. M. W. Hirsch, Systems of differential equations that are competitive or cooperative. II: Convergence almost everywhere, SIAM J. Math. Anal., 16 (1985), 423-439. MR.783970|(87a:58137)

14. M. W. Hirsch, Stability and convergence in strongly monotone dynamical systems, J. Reine Angew. Math., 383 (1988), 1-53. MR921986 (89c:58108)

15. M. Hirsch and H. L. Smith, Monotone dynamical systems, in: A. Canada, P. Drabek, A. Fonda (Eds.), Handbook of Differential Equations, Ordinary Differential Equations, second volume, Elsevier, Amsterdam, 2005. MR 2182759 (2006j:37017)

16. M. W. Hirsch, The dynamical systems approach to differential equations, Bull. Amer. Math. Soc., 11 (1984), 1-64. MR741723 (85m:58060) 
17. M. W. Hirsch, Positive equilibria and convergence in subhomogeneous monotone dynamics, pages 169-187, "Comparison Methods and Stability Theory" (X. Liu and D. Siegel, eds.), Lecture Notes in Pure and Applied Math, Vol. 162, Marcel Dekker, New York, 1994. MR 1291618 (95f:34090)

18. T. Krisztin and J. Wu, Monotone semiflows generated by neutral equations with different delays in neutral and retarded parts, Acta Math. Univ. Comeniane, 63 (1994), 207-220. MR.1319440 (96b:34097)

19. T. Krisztin and J. Wu, Asymptotic periodicity, monotonicity, and oscillation of solutions of scalar neutral functional differential equations, J. Math. Anal. Appl., 199 (1996), 502-525. MR:1383238 (97c:34163)

20. J. Jiang, On strongly monotone flows which are Liapunov stable, Acta Math. Sinica, 33 (1990), 786-790 (Chinese). MR1090628 (92e:34061)

21. J. Jiang, A note on a global stability theorem of M. W. Hirsch, Proc. Amer. Math. Soc., 112 (1991), 803-806. MR:1043411 (92b:58119)

22. J. Jiang, Periodic time dependent cooperative systems of differential equations with a first integral, Ann. Differential Equations, 8 (1992), 429-437. MR.1215988 (94b:34056)

23. J. Jiang, On the global stability of cooperative systems, Bull. London Math. Soc., 26 (1994), 455-458. MR.1308362 (95i:34089)

24. J. Jiang, Sublinear discrete-time order-preserving dynamical systems, Math. Proc. Camb. Phil. Soc., 119 (1996), 561-574. MR1357065 (96h:34090)

25. J. Jiang, Three- and four-dimensional cooperative systems with every equilibrium stable, J. Math. Anal. Appl., 188 (1994), 92-100. MR1301718 (95i:34049)

26. J. Jiang, Three-dimensional order-preserving discrete time dynamical systems with every fixed point stable, Commun. Appl. Nonlinear Anal., 2 (1995), 85-95. MR1343599 (96e:58085)

27. J. Jiang, On the analytic order-preserving discrete-time dynamical systems in $\mathbf{R}^{n}$ with every fixed point stable, J. London Math. Soc. (2), 53 (1996), 317-324. MR.1373063 (97h:58131)

28. J. Jiang, Periodic monotone systems with an invariant function, SIAM J. Math. Anal., 27 (1996), 1738-1744. MR1416516 (98h:34089)

29. J. Jiang and $\mathrm{X}$. Zhao, Convergence in monotone and uniformly stable skew-product semiflows with applications, J. Reine Angew. Math., 589 (2005), 21-55. MR2194677(2006k:37031)

30. J. Jiang and S. Yu, Stable cycles for attractors of strongly monotone discrete-time dynamical systems, J. Math. Anal. Appl., 202 (1996), 349-362. MR.1402605 (97e:58136)

31. H. Hu and J. Jiang, Translation-invariant monotone systems. I: Autonomous/periodic case, Nonlinear Anal.: Real World Appl., doi:10.016/j.nonrwa.2009.11.015.

32. J. Mierczyński, Strictly cooperative systems with a first integral, SIAM J. Math. Anal., 18 (1987), 642-646. MR883558 (88e:34093)

33. V. Muñoz-Villarragut, S. Novo and R. Obaya, Neutral functional differential equations with applications to compartmental systems, SIAM J. Math. Anal., 40 (2008), 1003-1028. MR 2452877 (2009i:34170)

34. F. Nakajima, Periodic time-dependent gross-substitute systems, SIAM J. Appl. Math., 36 (1979), 421-427. MR531605 (80c:93045)

35. S. Novo, R. Obaya and A. M. Sanz, Stability and extensibility results for abstract skew-product semiflows, J. Differential Equations, 235 (2007), 623-646. MR2317498(2008h:37018)

36. P. Poláčik, Convergence in smooth strongly monotone flows defined by semilinear parabolic equations, J. Differential Equations, 79 (1989), 89-110. MR.997611 (90f:58025)

37. P. Poláčik and I. Terešćák, Convergence to cycles as a typical asymptotic behavior in smooth strongly monotone discrete-time dynamical systems, Arch. Rational Mech. Anal., 116 (1992), 339-360. MR:1132766 (93b:58088)

38. W. Shen and X.-Q. Zhao, Convergence in almost periodic cooperative systems with a first integral, Proc. Amer. Math. Soc., 133 (2005), 203-212. MR2085171 (2005d:34076)

39. W. Shen and Y. Yi, Almost automorphic and almost periodic dynamics in skew-product semiflows, Memoirs of the American Mathematical Society, No. 647, Vol. 136, Providence, RI, 1998. MR1445493 (99d:34088)

40. H. L. Smith, Cooperative systems of differential equations with concave nonlinearities, Nonlinear Analysis, TMA, 10 (1986), 1037-1052. MR.857738(87k:58247)

41. H. L. Smith, Monotone Dynamical Systems, An Introduction to the Theory of Competitive and Cooperative Systems, Math. Surv. Monogr. 41, Amer. Math. Soc., Providence, RI, 1995. MR 1319817 (96c:34002) 
42. P. Takác̆, Convergence to equilibrium on invariant d-hypersurfaces for strongly increasing discrete-time semigroups, J. Math. Anal. Appl., 148 (1990), 223-244. MR1052057|(91d:58125)

43. P. Takáč, Asymptotic behavior of discrete-time semigroups of sublinear, strongly increasing mappings with applications to biology, Nonlinear Analysis, TMA, 14 (1990), 35-42. MR $1028245(90 \mathrm{j}: 47088)$

44. Y. Wang and X.-Q. Zhao, Convergence in monotone and subhomogeneous discrete dynamical systems on product Banach spaces, Bulletin of the London Math. Soc., 35 (2003), 681-688. MR 1989498 (2004c:35157)

45. J. Wu, Convergence in neutral equations with infinite delay arising from active compartmental systems, "World Congress of Nonlinear Analysts '92", Vols. I-IV (Tampa, FL, 1992), 13611369, de Gruyter, Berlin, 1996. MR1389170

46. J. Wu and H. I. Freedman, Monotone semiflows generated by neutral functional-differential equations with application to compartmental systems, Can. J. Math., 43 (1991), 1098-1120. MR 1138586 (92j:34141)

47. J. Wu, Convergence of monotone dynamical systems with minimal equilibria, Proc. Amer. Math. Soc., 106 (1989), 907-911. MR.1004632 (90j:58130)

Department of Mathematics, Tongui University, Shanghai 200029, People's Republic OF CHINA

E-mail address: hhxiao1@126.com

Mathematics and Science College, Shanghai Normal University, Shanghai 200234, People's Republic of China

E-mail address: jiangjf@shnu.edu.cn 